\title{
$\mathrm{ZnO}$ 掺杂对 $\mathrm{Ca}_{0.25}\left(\mathrm{Li}_{0.43} \mathrm{Sm}_{0.57}\right)_{0.75} \mathrm{TiO}_{3}$ 陶瓷烧结性能 和微波介电性能的影响
}

\author{
李海涛, 李谦, 间焉服, 许荣辉 \\ (河南科技大学 材料科学与工程学院, 洛阳 471003)
}

\begin{abstract}
摘 要: 采用溶胶-凝胶法制备 $\mathrm{Ca}_{0.25}\left(\mathrm{Li}_{0.43} \mathrm{Sm}_{0.57}\right)_{0.75} \mathrm{TiO}_{3}(\mathrm{CLST})$ 微波介质陶瓷纳米粉体, 研究了 $\mathrm{ZnO}$ 掺杂量和烧结 温度对 CLST $+x \mathrm{~mol} \% \mathrm{ZnO}$ 陶瓷烧结性能和微波介电性能的影响。XRD 分析结果表明: 随着 $\mathrm{ZnO}$ 掺杂量 $x$ 的增加, 陶瓷的晶体结构从正交相变为伪立方相, 并在 $x \geqslant 1.5$ 的样品中出现了杂相。CLST $+x \mathrm{~mol} \% \mathrm{ZnO}$ 陶瓷的致密化烧结 温度随 $x$ 的增加而降低, $x=1.0$ 的样品的致密化烧结温度比 $x=0$ 的降低了 $200{ }^{\circ} \mathrm{C}$ 。介电常数 $\varepsilon_{\mathrm{r}}$ 和频率品质因数 $Q f$ 随 $x$ 增加和烧结温度的升高具有最优值, 频率温度系数则单调降低。 $x=1.0$ 的样品在 $1100{ }^{\circ} \mathrm{C}$ 烧结时具有优异的综合性 能: $\rho=4.85 \mathrm{~g} / \mathrm{cm}^{3}, \varepsilon_{\mathrm{r}}=102.8, Q f=5424 \mathrm{GHz}, \tau_{f}=-8.2 \times 10^{-6} /{ }^{\circ} \mathrm{C}$ 。表明 $\mathrm{ZnO}$ 掺杂的 CLST 陶瓷是一种很有发展潜力的 微波介质陶瓷。
\end{abstract}

关 键 词: 溶胶-凝胶; CLST; 微波介质陶瓷; 致密化烧结

中图分类号: TQ174 文献标识码: A

\section{Effect of ZnO-doping on Sinterability and Microwave Dielectric Property of $\mathrm{Ca}_{0.25}\left(\mathrm{Li}_{0.43} \mathrm{Sm}_{0.57}\right)_{0.75} \mathrm{TiO}_{3}$ Ceramics}

\author{
LI Hai-Tao, LI Qian, YAN Yan-Fu, XU Rong-Hui
}

(School of Materials Science and Engineering, Henan University of Science and Technology, Luoyang 471003, China)

\begin{abstract}
The}^{\mathrm{Ca}} \mathrm{Ca}_{0.25}\left(\mathrm{Li}_{0.43} \mathrm{Sm}_{0.57}\right)_{0.75} \mathrm{TiO}_{3}$ (CLST) ceramic nano-powder was prepared by Sol-Gel method. The influences of $\mathrm{ZnO}$ doping content and sintering temperature on sintering behavior and microwave dielectric properties of CLST ceramics were investigated. A phase transition from orthorhombic to pseudo cubic symmetry was identified by XRD analysis, and a impure phase was found in the $x \geqslant 1.5$ samples. The densification sintering temperature of the $\mathrm{CLST}+x \mathrm{~mol} \% \mathrm{ZnO}$ ceramics decrease with increasing $\mathrm{ZnO}$ doping content, and it reduces from $1300^{\circ} \mathrm{C}$ to $1100^{\circ} \mathrm{C}$ when the amount of $\mathrm{ZnO}$ doping increase from $x=0$ to $x=1.0$. The results also reveal that both the dielectric constant $\varepsilon_{\mathrm{r}}$ and the quality factor $Q f$ possess a peak value with increasing $\mathrm{ZnO}$ doping content or sintering temperature, whereas the frequency temperature coefficient $\tau_{\mathrm{f}}$ decrease monotonously. The $x=1.0$ sample sintered at $1100^{\circ} \mathrm{C}$ for $2 \mathrm{~h}$ has optimal microwave dielectric properties: $\rho=4.85 \mathrm{~g} / \mathrm{cm}^{3}, \varepsilon_{\mathrm{r}}=102.8, Q f=5424 \mathrm{GHz}, \tau_{f}=-8.2 \times 10^{-6} /{ }^{\circ} \mathrm{C}$, respectively, which indicates that the CLST ceramic doped with $1.0 \mathrm{~mol} \% \mathrm{ZnO}$ is a promising microwave dielectric ceramic.
\end{abstract}

Key words: Sol-Gel; CLST; microwave dielectric ceramic; densification sintering temperature 
近年来, 以微波应用为代表的通讯技术发展迅 猛, 使得通讯设备趋向小型、轻量、高频、多功能、 高性能方向发展。这对以微波介质陶瓷材料为基础 的微波电路元器件提出了更高要求。低成本、低能 耗、可以与高导电率的贱金属电极材料(如银、铜、 镍等)共烧成为微波介质陶瓷研究和发展的方向 ${ }^{[1-5]}$ 。 $\mathrm{MO}-\mathrm{Li}_{2} \mathrm{O}-\mathrm{Ln}_{2} \mathrm{O}_{3}-\mathrm{TiO}_{2}(\mathrm{M}=\mathrm{Ba} 、 \mathrm{Ca}, \mathrm{Ln}=\mathrm{La} 、 \mathrm{Pr} 、 \mathrm{Sm}$ 、 $\mathrm{Eu} 、 \mathrm{Gd}$ 等) 是上世纪 90 年代初提出的一个微波介 质陶瓷体系, 具有较高的介电常数 $\left(\varepsilon_{\mathrm{r}} \approx 100\right)$, 适中的 介电损耗, 有望成为小型化高频通讯设备的介质材 料, 引起了人们的广泛兴趣 ${ }^{[6-8]}$ 。Ezaki 等 ${ }^{[9]}$ 首先报道 了非化学计量比的 $\mathrm{CaO}_{2}-\mathrm{Li}_{2} \mathrm{O}-\mathrm{Sm}_{2} \mathrm{O}_{3}-\mathrm{TiO}_{2}$ (摩尔比 为 16:9:12:63) 陶瓷具有较好的温度稳定性、较高的 介电常数和适中的品质因数 $(Q f)$, 但此类陶瓷的烧 结温度高达 $1300^{\circ} \mathrm{C}$, 很难与熔点较低的相对廉价的 金属 $\mathrm{Ag}\left(961^{\circ} \mathrm{C}\right) 、 \mathrm{Cu}\left(1064^{\circ} \mathrm{C}\right)$ 共烧, 因此限制了其 发展应用。Huang 等 ${ }^{[10]}$ 研究了烧结温度 (1250 $1370^{\circ} \mathrm{C}$ ) 对 CLST 陶瓷的微波性能的影响, 发现烧结 温度对陶瓷的微观形貌和介电性能都有较大影响, 经 $1310^{\circ} \mathrm{C}$ 烧结的陶瓷样品具有优异的介电性能: $Q f=5700 \mathrm{GHz}, \varepsilon_{\mathrm{r}}=108$; 经 $1350^{\circ} \mathrm{C}$ 烧结的样品, $\tau_{\mathrm{f}}=8 \times$ $10^{-6} /{ }^{\circ} \mathrm{C}$, 介电常数和品质因数有所降低。Yoon 等 ${ }^{[11]}$ 通过在 $\left(\mathrm{Ca}_{0.275} \mathrm{Li}_{0.25} \mathrm{Sm}_{0.4}\right) \mathrm{TiO}_{3}$ 中掺杂 $\mathrm{B}_{2} \mathrm{O}_{3}-\mathrm{Li}_{2} \mathrm{O}$ 来 提高陶瓷的烧结性能, 当 $\mathrm{B}_{2} \mathrm{O}_{3}-\mathrm{Li}_{2} \mathrm{O}$ 掺杂量达到 $5 \mathrm{wt} \%$ 时, 陶瓷体的密度达到理论密度的 $99 \%$, 并且 烧结温度也有所降低, 不过仍然在 $1200^{\circ} \mathrm{C}$ 以上。 $\mathrm{Gu}$ 等 ${ }^{[7]}$ 通过添加 $\mathrm{BaCu}\left(\mathrm{B}_{2} \mathrm{O}_{5}\right)$ 使 $\mathrm{CaO}_{2}-\mathrm{Li}_{2} \mathrm{O}-\mathrm{Sm}_{2} \mathrm{O}_{3}-\mathrm{TiO}_{2}$ 的烧结温度降到 $1050{ }^{\circ} \mathrm{C}$, 但其微波介电性能较低: $\tan \delta=0.0212, \varepsilon_{\mathrm{r}}=81.4$ 。因此, 如何在保持较高微波介 电性能的基础上, 降低 CLST 陶瓷的烧结温度成为 人们研究的焦点。

目前, 降低 CLST 陶瓷烧结温度的方法主要有 以下几种: 选择固有烧结温度低的陶瓷基体(如含 $\mathrm{Bi}$ 的陶瓷); 选择低熔点的化合物 (如 $\mathrm{CuO} 、 \mathrm{~B}_{2} \mathrm{O}_{3}$ 等) 或玻璃相(如 $\mathrm{ZnO}-\mathrm{B}_{2} \mathrm{O}_{3}$ 系玻璃、 $\mathrm{SiO}_{2}-\mathrm{B}_{2} \mathrm{O}_{3}$ 系玻璃 等)作为助烧剂; 利用湿化学法制备纳米级陶瓷粉 体等。本工作采用溶胶-凝胶法制备纳米 CLST 陶瓷 粉体, 以 $\mathrm{ZnO}$ 为烧结助剂, 研究了 $\mathrm{ZnO}$ 掺杂量及烧 结温度对陶瓷烧结性能及微波介电性能的影响。

\section{1 实验方法}

\section{1 样品制备}

以分析纯的 $\mathrm{CaCO}_{3}$ (纯度> $99 \%$ )、 $\mathrm{Li}_{2} \mathrm{CO}_{3}$ (纯度> 99.9\%)、 $\mathrm{Sm}_{2} \mathrm{O}_{3}$ (纯度>99.9\%)、 $\mathrm{C}_{16} \mathrm{H}_{36} \mathrm{O}_{4} \mathrm{Ti}$ (纯度> $99 \%$ ) 及 $\mathrm{ZnO}$ (纯度 $>99.5 \%$ ) 为原料。按化学式
$\mathrm{Ca}_{0.25}\left(\mathrm{Li}_{0.43} \mathrm{Sm}_{0.57}\right)_{0.75} \mathrm{TiO}_{3}$ 进行配料, 先将 $\mathrm{Sm}_{2} \mathrm{O}_{3}$ 溶 解于硝酸中配成溶液 $1 ; \mathrm{CaCO}_{3} 、 \mathrm{Li}_{2} \mathrm{CO}_{3}$ 溶解于去 离子水中配成溶液 2 ; 钛酸四丁酯缓慢注入硝酸, 边注入边摚拌直到完全溶解, 形成溶液 3 。三种溶液 配好后, 再将溶液 1 和 2 在 $80^{\circ} \mathrm{C}$ 电磁摚拌 $1 \mathrm{~h}$ 混合 均匀, 然后加入适量的柠檬酸, 调节 $\mathrm{pH}=6$ 。将混合 后的溶液和溶液 3 混合均匀, 调节 $\mathrm{pH}=5 \sim 6,100^{\circ} \mathrm{C}$ 下 电磁搅拌 $4 \mathrm{~h}$ 反应生成溶胶。充分反应后的溶胶在 $80^{\circ} \mathrm{C}$ 干燥成为凝胶。凝胶在红外干燥器中脱水干燥, 脱水后的干凝胶在空气中加少量乙醇点燃, 变成黑 色灰粉, 燃烧后的产物在 900 950 ${ }^{\circ} \mathrm{C}$ 㷽烧 $4 \mathrm{~h}$ 合成 纳米 $\mathrm{Ca}_{0.25}\left(\mathrm{Li}_{0.43} \mathrm{Sm}_{0.57}\right)_{0.75} \mathrm{TiO}_{3}$ 陶瓷粉体, 合成的粉 体加入适量的聚乙二醇用超声波分散 $10 \mathrm{~min}$ 。

按化学式 $\mathrm{Ca}_{0.25}\left(\mathrm{Li}_{0.43} \mathrm{Sm}_{0.57}\right)_{0.75} \mathrm{TiO}_{3}+x \mathrm{~mol} \%$ $\mathrm{ZnO}(\mathrm{CLST}+x \mathrm{~mol} \% \mathrm{ZnO})(x=0 、 0.5 、 1.0 、 1.5 、 2.0)$ 精确配料。称好的料放入氧化锆球磨罐中, 用无水 乙醇为球磨介质, 在行星式球磨机中球磨 $6 \mathrm{~h}$ 。烘干 后的粉体加入 $5 \mathrm{wt} \%$ 的 PVA 做为粘结剂造粒, 干压 成型，制成 $\phi 10 \mathrm{~mm} \times 6 \mathrm{~mm}$ 的圆柱体。制成的生坏 分别在不同温度 $\left(1050^{\circ} \mathrm{C} 、 1100^{\circ} \mathrm{C} 、 1150^{\circ} \mathrm{C} 、 1200^{\circ} \mathrm{C}\right.$ 和 $1250^{\circ} \mathrm{C}$ )下烧结 $2 \mathrm{~h}$, 制备陶瓷样品。

\section{2 性能检测}

用阿基米德排水法测试陶瓷样品的密度, 用 $\mathrm{X}$ 射线衍射仪(X-ray diffraction, XRD, Rigaku RAD-B system, Japan)分析其相结构, 用扫描电子显微镜 (scanning electron microscope, SEM, JSM-6460LV, JEOL, Japan) 观察其显微结构, 用透射电子显微境 (transmission electron microscope, TEM, Philips CM200, Netherlands)分析粉体颗粒的大小和形貌。 用网络分析仪 (network analyzer, PNA-L, Agilent, America)测试样品的微波介电性能, 谐振模式为 TE011。在 25 80 ${ }^{\circ} \mathrm{C}$ 采用谐振腔法测量谐振频率温度 系数 $\tau_{\mathrm{f}}$, 其计算公式为:

$$
\tau_{\mathrm{f}}=\frac{f(80)-f(25)}{f(25) *(80-25)}
$$

式中: $f(25)$ 为 $25^{\circ} \mathrm{C}$ 时的谐振频率, $f(80)$ 为 $80^{\circ} \mathrm{C}$ 时 的谐振频率。

\section{2 结果与讨论}

\section{1 纳米粉体形貌}

图 1 是 $950^{\circ} \mathrm{C}$ 煅烧 $4 \mathrm{~h}$ 的溶胶一凝胶法制备的纳 米 CLST 陶瓷粉体的 TEM 照片。由图 1(a)可以看出 粉体颗粒尺寸在 30 40 nm 之间, 颗粒大小均匀, 但 团聚现象比较严重。这主要是由于前驱体在干燥和 

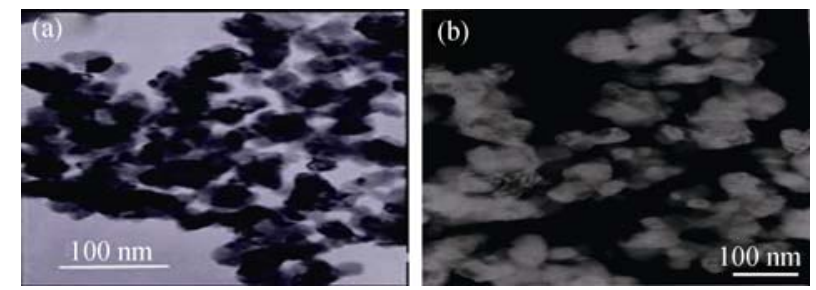

图 $1950{ }^{\circ} \mathrm{C}$ 裧烧 $4 \mathrm{~h} \mathrm{CLST}$ 陶瓷粉体颗粒的 TEM 照片

Fig. 1 TEM micrographs of CLST ceramic powders calcinated at $950{ }^{\circ} \mathrm{C}$ for $4 \mathrm{~h}$ before (a) and after (b) being dispersed

煆烧过程中，相邻颗粒表面的羟基氢键转化成强度 更高的桥氧键而使颗粒形成硬团聚; 另外, 水分脱 除过程中, 前驱体凝胶网络之间产生的毛细管力, 使颗粒收紧重排, 也是造成颗粒团聚的一个重要原 因。图 1(b) 为经 $0.5 \mathrm{wt} \%$ 的聚乙二醇分散后的纳米 CLST 陶瓷粉体的 TEM 照片, 与图 1(a)相比, 粉体 颗粒分散较均匀, 团聚程度降低。由于纳米颗粒比 表面积较大, 活性较强, 因此对其进行分散处理至 关重要。只有这样才能真正发挥其颗粒较小的优势, 增加其烧结活性，降低烧结温度。

\section{2 陶瓷的相结构}

图 2 为 $\mathrm{CLST}+x \mathrm{~mol} \% \mathrm{ZnO}$ 陶瓷的 XRD 图谱, 由图可知，当 $x<1.5$ 时，陶瓷 CLST 特征衍射峰在衍 射角为 $47^{\circ}$ 附近分裂成 2 个, 陶瓷主晶相为正交钙 钛矿结构(PDF 78-1013)。当 $x \geqslant 1.5$ 时, $47^{\circ}$ 附近的特 征衍射峰合并成一个峰, 陶瓷主晶相变为伪立方钙 钛矿结构 ${ }^{[12]}$, 在 $30^{\circ}$ 附近出现微弱的第二相衍射峰, 经 Jade 软件分析可知, 第二相为 $\mathrm{Zn}_{2} \mathrm{Ti}_{3} \mathrm{O}_{8}(\mathrm{PDF}$ 78-1013), 且随着掺杂量 $x$ 的增加, 第二相衍射峰逐 渐增强。表明当 $x<1.5$ 时, $\mathrm{Zn}^{2+}$ 进入 CLST 陶瓷主晶 相晶格形成均一、稳定的固溶体; $x \geqslant 1.5$ 时, $\mathrm{Zn}^{2+}$ 在 CLST 晶格中的固溶度已达到固溶极限, 多余的 $\mathrm{Zn}^{2+}$ 和 $\mathrm{Ti}^{4+}$ 结合生成第二相 $\mathrm{Zn}_{2} \mathrm{Ti}_{3} \mathrm{O}_{8}$; 过多 $\mathrm{Zn}^{2+}$ 的 进入使陶瓷晶格结构发生畸变, 晶相由正交相变为

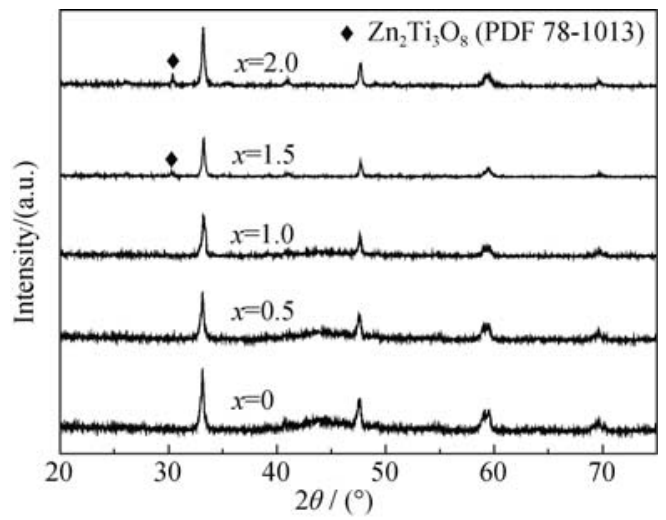

图 $2 \mathrm{CLST}+x \mathrm{~mol} \% \mathrm{ZnO}$ 陶瓷的 XRD 图谱

Fig. 2 XRD patterns of the CLST $+x$ mol $\% \mathrm{ZnO}$ ceramics
伪立方相。由图还可以看出，随掺杂量 $x$ 的增大，陶 瓷主晶相衍射峰逐渐向高角度偏移, 由布拉格方程 $2 d \sin \theta=\lambda$ 可知陶瓷的晶面间距缩短，晶胞体积缩小。 这是由于 $\mathrm{Zn}^{2+}$ 与 $\mathrm{Ca}^{2+}$ 具有相同的化合价和电性能, 故 $\mathrm{Zn}^{2+}$ 进入晶格的 $\mathrm{A}$ 位取代 $\mathrm{Ca}^{2+}$, 而 $\mathrm{Zn}^{2+}$ 半径 $(0.074 \mathrm{~nm})$ 比 $\mathrm{Ca}^{2+}$ 的半径 $(0.099 \mathrm{~nm})$ 小, 因此使晶面 间距变短, 晶胞体积收缩。

\section{3 陶瓷的致密度}

图 3 为不同 $\mathrm{ZnO}$ 掺杂量的陶瓷样品经 1050 、 $1100 、 1150 、 1200$ 和 $1250^{\circ} \mathrm{C}$ 温度烧结的体积密度曲 线。由图 3 可以看出，当 $x=0$ 时，即不掺杂 $\mathrm{ZnO}$ 陶 瓷的体积密度随烧结温度的升高而增大，据报道 CLST 陶瓷的致密化烧结温度为 $1300^{\circ} \mathrm{C}$, 已超出了 本研究所考查的温度范围上限 $1250^{\circ} \mathrm{C}$; 当 $x=2.0$ 时, 陶瓷的体积密度则随烧结温度的升高而减小，说明 陶瓷的致密化烧结温度小于本研究所考查的烧结温 度下限 $1050^{\circ} \mathrm{C}$; 当 $x=0.5 \sim 1.5$ 时, 陶瓷的体积密度则 随着烧结温度的升高先增大后减小, 当 $x=0.5$ 时, $1200^{\circ} \mathrm{C}$ 烧结陶瓷的密度达最大值 $4.69 \mathrm{~g} / \mathrm{cm}^{3}$; 当 $x=1.0$ 和 $x=1.5$ 时, $1100^{\circ} \mathrm{C}$ 烧结陶瓷的密度分别达最大值 4.85 和 $4.89 \mathrm{~g} / \mathrm{cm}^{3}$, 总的来说陶瓷的致密化烧结温度 随 $\mathrm{ZnO}$ 掺杂量的增大而降低。这说明 $\mathrm{ZnO}$ 发挥了助 烧作用, 使 CLST 陶瓷的致密化烧结温度从 $1300^{\circ} \mathrm{C}$ 降 到 $1050^{\circ} \mathrm{C}$ 甚至更低。当烧结温度高于陶瓷的致密化烧 结温度后, 更高的烧结温度并不能提高陶瓷的致密度, 反而会因个别晶粒过分长大，而使陶瓷晶粒变得不均 匀, 从而降低其体积密度 ${ }^{[13-15]}$ 。

\section{4 陶瓷的显微结构}

图 4 为不同温度烧结的 CLST $+1.0 \mathrm{~mol} \% \mathrm{ZnO}$ 陶瓷断口 SEM 照片，由图可以看出，在 $1050^{\circ} \mathrm{C}$ 温度 烧结时晶粒较小且不均匀, 并有较多气孔, 这是因 为烧结温度较低，晶粒没有发育完全的缘故，如图

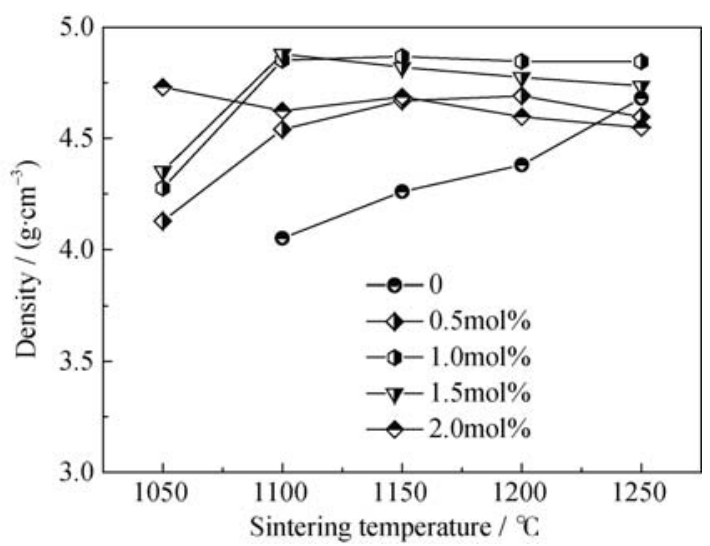

图 $3 \mathrm{CLST}+x \mathrm{~mol} \% \mathrm{ZnO}$ 陶瓷的密度-烧结温度曲线

Fig. 3 Bulk density vs sintering temperature of CLST $+x \mathrm{~mol} \%$ $\mathrm{ZnO}$ ceramics 


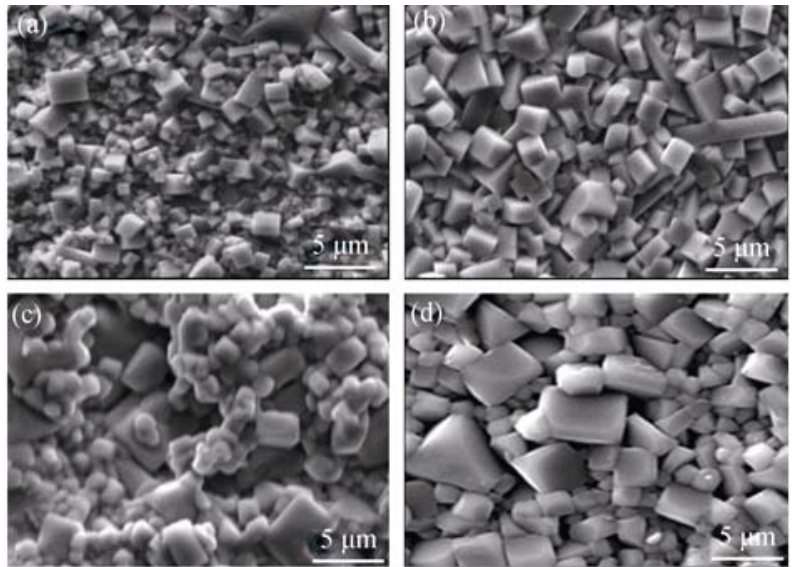

图 4 不同烧结温度下 CLST+1.0mol\% $\mathrm{ZnO}$ 陶瓷断口 $\mathrm{SEM}$ 照片

Fig. 4 SEM photographs of CLST $+1.0 \mathrm{~mol} \% \mathrm{ZnO}$ ceramics sintered at different temperatures

(a) $1050^{\circ} \mathrm{C}$; (b) $1110^{\circ} \mathrm{C}$; (c) $1150^{\circ} \mathrm{C}$; (d) $1200^{\circ} \mathrm{C}$

4(a)所示; 当烧结温度为 $1100^{\circ} \mathrm{C}$ 时，陶瓷致密且粒 径均匀, 晶粒发育较好, 粒径约为 2 3 $\mu \mathrm{m}$ (图 4(b)); 当烧结温度升到 $1150^{\circ} \mathrm{C}$ 时, 由于温度较高而使某些 晶粒出现异常长大现象, 陶瓷的粒径差别较大, 较 大的粒径达到 4 5 $\mu \mathrm{m}$, 较小的粒径约为 1 2 $\mu \mathrm{m}$ (图 4(c)); 当烧结温度升高到 $1200^{\circ} \mathrm{C}$ 时, 异常长大的晶 粒数量进一步增多, 粒径也进一步增大, 较大的粒 径达到 5 6 $4 \mu \mathrm{m}$, 较小的粒径约为 $2 \sim 3 \mu \mathrm{m}$, 由于晶粒 分布不均匀, 晶界间有较多气孔存在, 导致陶瓷体 积密度下降 (图 4(d))。实验中的烧结时间只有 $2 \mathrm{~h}$, 在如此短的烧结时间内陶瓷粒径增长的如此快的现 象可用液相烧结来解释 ${ }^{[13]} \mathrm{ZnO}$ 的熔点在 $1500^{\circ} \mathrm{C}$ 以 上, 液相的成分不可能是单纯的 $\mathrm{ZnO}$, 有可能是 $\mathrm{ZnO}$ 和 $\mathrm{Li}_{2} \mathrm{O}$ 或 $\mathrm{ZnO} 、 \mathrm{Li}_{2} \mathrm{O}$ 和 $\mathrm{CaO}$ 的混合物。烧结 前期液相降低晶粒长大的临界驱动能, 促进晶粒的 发育; 烧结后期液相进入主晶相晶格补偿烧结过程 中形成的空位。在烧结剂量一定时, 生成的液相量 只与烧结温度有关。当烧结温度较低, 生成的液相 量较少时, 陶瓷晶粒发育不完全, 如图 4(a)所示; 当烧结温度适宜, 生成适量的液相, 陶瓷晶粒完全 发育, 显微结构较好, 此时的烧结温度就是该陶瓷 的致密化烧结温度, 如图 4(b)所示; 当烧结温度高 于致密化烧结温度时, 生成较多的液相, 使得陶瓷 的部分晶粒出现异常长大的现象, 粒径呈现较大差 异, 导致致密度下降, 显微结构变差, 如图 4(c)、(d) 所示。

\section{5 陶瓷的介电性能}

图 5 为 CLST 陶瓷样品在 $1100^{\circ} \mathrm{C}$ 烧结时, 介电常 数 $\varepsilon_{\mathrm{r}}$ 、频率品质因数 $Q f$ 和频率温度系数 $\tau_{\mathrm{f}}$ 随着 $\mathrm{ZnO}$
掺杂量 $x$ 的变化曲线。由图 5 可以看出, $\varepsilon_{\mathrm{r}}$ 和 $Q f$ 都 随 $x$ 的增加先增大后减小, $\varepsilon_{\mathrm{r}}$ 在 $x=1.0$ 时达到最大值 102.8, $Q f$ 在 $x=1.5$ 时达到最大值 $5545 \mathrm{GHz}$ 。陶瓷的 介电常数受致密度、掺杂元素及第二相等多种因素 的影响。一方面, ZnO 做为助烧剂能够促进 CLST 陶瓷的烧结, 降低致密化烧结温度, 提高其致密度, 使介电常数增大; 另一方面, 从离子半径和离子电 荷方面分析, Zn 掺杂主要进入 CLST 陶瓷的 A 位取 代 $\mathrm{Ca}^{2+}$ 的位置, 由于 $\mathrm{Zn}^{2+}$ 半径小于 $\mathrm{Ca}^{2+}$ 半径, 两者 的半径差会导致 CLST 陶瓷的晶胞体积减小, 引起 八面体的收缩，使外场作用下 $\mathrm{Ti}$ 离子可移动的空间 减小, 从而使陶瓷的介电常数减小 ${ }^{[16]}$; 另外, 杂相 也是使介电常数减小的因素之一。因此, $\varepsilon_{\mathrm{r}}$ 在 $x=1.0$ 时达到最大值是上述几方面因素共同作用的结果。

CLST 陶瓷的 $Q f$ 受致密度、晶粒尺寸、气孔及缺陷 和相组成等多种因素的影响, 其变化趋势是这几种 因素共同作用的结果。 $\tau_{\mathrm{f}}$ 随 $x$ 的增加而减小, 从 $x=0$ 时的 $20.6 \times 10^{-6} /{ }^{\circ} \mathrm{C}$ 到 $x=2.0$ 时的 $-15.4 \times 10^{-6} /{ }^{\circ} \mathrm{C}$ 。不 掺杂 $\mathrm{ZnO}$ 的 CLST 陶瓷样品 $\tau_{\mathrm{f}}$ 为正值, 添加 $\mathrm{ZnO}$ 后 $\tau_{\mathrm{f}}$ 向负方向移动，有效地调节了 CLST 陶瓷的正温度 系数。适当的 $\mathrm{ZnO}$ 掺杂量可获得 $\tau_{\mathrm{f}}$ 接近于 0 的 CLST 微波介质陶瓷，这与我们的前期报道相一致 ${ }^{[2]}$ 。

图 6 为掺杂量 $x=1.0$ 的 CLST 陶瓷样品的介电 常数 $\varepsilon_{\mathrm{r}}$ 、频率品质因数 $Q f$ 、频率温度系数 $\tau_{\mathrm{f}}$ 随烧结 温度的变化曲线。由图 6 可以看出, $\varepsilon_{\mathrm{r}}$ 和 $Q f$ 都随烧

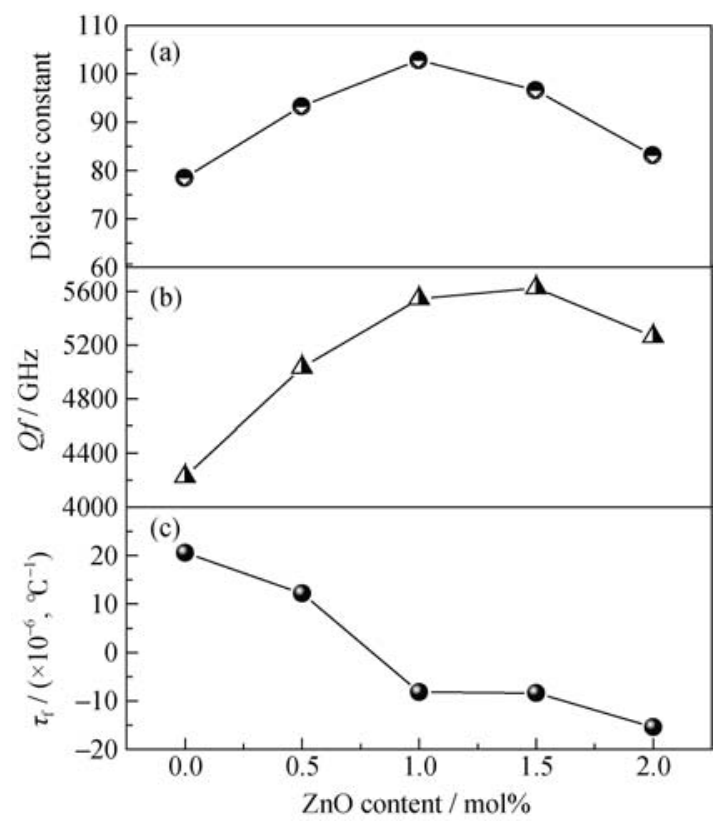

图 5 不同 $\mathrm{ZnO}$ 掺杂量 CLST 陶瓷的介电常数(a)、频率品质 因数(b)和频率温度系数(c)

Fig. 5 Dielectric constant $\left(\varepsilon_{\mathrm{r}}\right)(\mathrm{a})$, quality factor $(Q f)(\mathrm{b})$ and frequency temperature coefficient $\left(\tau_{\mathrm{f}}\right)(\mathrm{c})$ of $\mathrm{CLST}+x \mathrm{~mol} \% \mathrm{ZnO}$ ceramics 


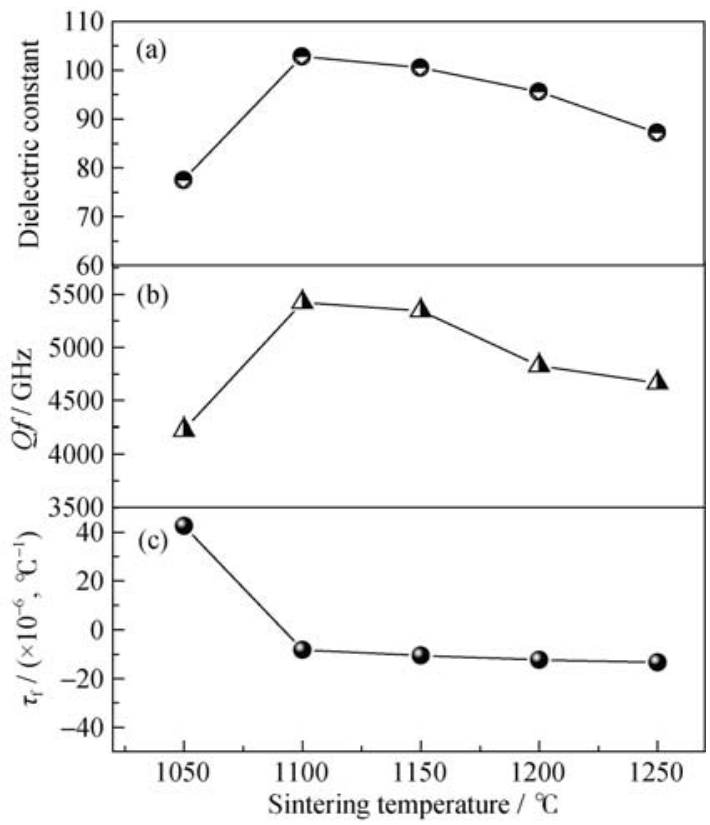

图 $6 \mathrm{CLST}+1.0 \mathrm{~mol} \% \mathrm{ZnO}$ 陶瓷的介电常数(a)、频率品质因 数(b)和频率温度系数(c)随烧结温度的变化曲线

Fig. 6 Dielectric constant $\left(\varepsilon_{\mathrm{r}}\right)(\mathrm{a})$, quality factor $(Q f)(\mathrm{b})$ and frequency temperature coefficient $\left(\tau_{\mathrm{f}}\right)(\mathrm{c})$ of CLST $+1.0 \mathrm{~mol} \%$ $\mathrm{ZnO}$ ceramics sintered at different temperatures

结温度的升高先增大后减小, $1100^{\circ} \mathrm{C}$ 烧结时分别达到 最大值 102.8 和 $5424 \mathrm{GHz}$ 。根据上面的分析, 当掺杂 量 $x$ 相同时, $\varepsilon_{\mathrm{r}}$ 和 $Q f$ 主要受致密度的影响。结合图 3 和图 4, $x=1.0$ 的样品在 $1100^{\circ} \mathrm{C}$ 烧结时密度最大, 粒径 比较均匀, 气孔较少。因此, 样品在 $1100^{\circ} \mathrm{C}$ 烧结时 $\varepsilon_{\mathrm{r}}$ 和 $Q f$ 达到最优值。 $\tau_{\mathrm{f}}$ 随烧结温度的升高而减小, 当 烧结温度高于 $1100^{\circ} \mathrm{C}$ 后变化比较平缓。这是由于随 烧结温度升高, 陶瓷中液相增多, 而液相的频率温度 系数 $\tau_{\mathrm{f}}$ 为负值, 因此 $\tau_{\mathrm{f}}$ 随烧结温度的升高而减小, 这 与李月明等报道结果相一致 ${ }^{[17]}$ 。

\section{3 结论}

1) 利用溶胶一凝胶法成功合成了 CLST 陶瓷纳 米粉体, 加入聚乙二醇超声分散能很好地解决 CLST 纳米陶瓷粉体的团聚问题。

2) 随 $\mathrm{ZnO}$ 掺杂量的增加和烧结温度的升高, CLST 陶瓷的介电常数、频率品质因数都呈现先增 大后减小的趋势, 频率温度系数则不断减小。

3) $1100^{\circ} \mathrm{C}$ 烧结的掺杂量为 $x=1.0$ 的样品具有优 异的介电性能: $\varepsilon_{\mathrm{r}}=102.8, Q f=5424 \mathrm{GHz}, \tau_{f}=-8.2 \times$ $10^{-6} /{ }^{\circ} \mathrm{C}$ 。

4) 添加适量的 $\mathrm{ZnO}$ 能够调节 CLST 陶瓷的正 温度系数接近 0 , 增加了该类陶瓷的温度稳定性。
参考文献:

[1] DONG JIN-WEN, XU JIAN-MEI, ZHANG DE, et al. The low-temperture sintering and microwave dielectric properties of $\mathrm{Mg}_{3}\left(\mathrm{VO}_{4}\right)_{2}-\mathrm{BiNbO}_{4}$ composite ceramics. J. Chin. Ceram. Soc., 2010, 38(3): 381-386.

[2] LU YIN, LI HAI-TAO, LI QIAN, et al. Microstructure and microwave dielectric properties of $\mathrm{ZnO}-\mathrm{B}_{2} \mathrm{O}_{3}$ added $\left(\mathrm{Ca}_{0.254} \mathrm{Li}_{0.19} \mathrm{Sm}_{0.14}\right) \mathrm{TiO}_{3}$ ceramics. Rare Metals, 2010, 29(3): 243-247.

[3] LIN XU-PING, MA JING-TAO, ZHANG BAO-QING, et al. Microwave dielectric properties of low-temperture fired $\mathrm{Zn}_{3} \mathrm{Nb}_{2} \mathrm{O}_{8}$ ceramics with $\mathrm{CuO}-\mathrm{V}_{2} \mathrm{O}_{5}-\mathrm{Bi}_{2} \mathrm{O}_{3}$. J. Chin. Ceram. Soc., 2010, 38(8): 1388-1391.

[4] MI YUE-AN, LI EN-ZHU, YU SHAO-YANG, et al. Effect of $\mathrm{Li}_{2} \mathrm{O}-\mathrm{B}_{2} \mathrm{O}_{3}-\mathrm{SiO}_{2}$ glass on low temperature sintering and microwave dielectric properties of M-phase $\mathrm{Li}_{1.0} \mathrm{Nb}_{0.6} \mathrm{Ti}_{0.5} \mathrm{O}_{3}$ ceramics. Electronic Components and Materials, 2014, 33(2): 68-71.

[5] YOU H W, KOO S M, HA J G, et al. Microstructure and dielectric properties of $\mathrm{Li}_{2} \mathrm{CO}_{3}$ doped $0.7(\mathrm{Ba}, \mathrm{Sr}) \mathrm{TiO}_{3}-0.3 \mathrm{MgO}$ ceramics. Current Applied Physics, 2009, 9: 875-879.

[6] LI YUE-MING, JIN YUN-HAI, SHEN ZONG-YANG, et al. Effects of composite sintering additives on properties of CSLST microwave dielectric ceramics. Journal of Synthetic Crystals, 2012, 41(5): $1221-1226$.

[7] GU YONG-JUN, HUANG JIN-LIANG, WANG YING, et al. Low temperature firing of $\mathrm{CaO}-\mathrm{Li}_{2} \mathrm{O}-\mathrm{Sm}_{2} \mathrm{O}_{3}-\mathrm{TiO}_{2}$ ceramics with $\mathrm{BaCu}\left(\mathrm{B}_{2} \mathrm{O}_{5}\right)$ addition. Solid State Communications, 2009, 149 (13-14) : 555-558.

[8] CHO J Y, YOON K H, KIM E S, et al. Microwave dielectric properties of layered $\mathrm{Mg}_{0.93} \mathrm{Ca}_{0.07} \mathrm{TiO}_{3}-\left(\mathrm{Ca}_{0.3} \mathrm{Li}_{0.14} \mathrm{Sm}_{0.42}\right) \mathrm{TiO}_{3}$ ceramics. Materials Chemistry and Physics, 2003, 79: 239-242.

[9] EZAKI K, BABA Y, TAKHASHI H, et al. Microwave dielectric properties of $\mathrm{CaO}-\mathrm{Li}_{2} \mathrm{O}-\mathrm{Ln}_{2} \mathrm{O}_{3}-\mathrm{TiO}_{2}$ Ceramics. Jpn. J. Appl. Phys., 1993, 32: 4319-4322.

[10] HUANG C L, TSAI J T, LI J L. Microwave dielectric properties of $(1-x) \mathrm{CaO}-x \mathrm{BaO}-\mathrm{Li}_{2} \mathrm{O}-(1-y) \mathrm{Sm}_{2} \mathrm{O}_{3}-y \mathrm{Nd}_{2} \mathrm{O}_{3}-\mathrm{TiO}_{2}$ ceramics system. Journal of Material Science, 2000, 35: 4901-4905.

[11] YOON K H, PARK M S, CHO J Y, et al. Effect of $\mathrm{B}_{2} \mathrm{O}_{3}-\mathrm{Li}_{2} \mathrm{O}$ on microwave dielectric properties of $\left(\mathrm{Ca}_{0.275} \mathrm{Sm}_{0.4} \mathrm{Li}_{0.25}\right) \mathrm{TiO}_{3}$ ceramics. J. Eur. Ceram. Soc., 2003, 23(14): 2423-2427.

[12] WANG K, LI J F. Analysis of crystallographic evolution in $(\mathrm{Na}, \mathrm{K}) \mathrm{NbO}_{3}$-based lead-free piezoceramics by X-ray diffraction. Appl. Phys. Lett., 2007, 91: 262902-1-3.

[13] PARK H Y, CHO K H, NAHM S, et al. Microstructure and piezoelectric properties of the $(1-x)\left(\mathrm{Na}_{0.5} \mathrm{~K}_{0.5}\right)-\mathrm{NbO}_{3}-x \mathrm{CaTiO}_{3}$ lead-free ceramics. J. Appl. Phys., 2007, 102: 124101.

[14] SEO I T, CHO K H, PARK H Y, et al. Effect of $\mathrm{CuO}$ on the sintering and piezoelectric properties of $0.95\left(\mathrm{Na}_{0.5} \mathrm{~K}_{0.5}\right) \mathrm{NbO}_{3}$ $-0.05 \mathrm{SrTiO}_{3}$ lead-free piezoelectric ceramics. J. Am. Ceram. Soc., 2008, 91(12): 3955-3960.

[15] LI HAI-TAO, ZHANG BO-PING, CUI MIN, et al. Microstructure, crystalline phase, and electrical properties of $\mathrm{ZnO}$-added $\mathrm{Li}_{0.06}\left(\mathrm{Na}_{0.535} \mathrm{~K}_{0.4}\right)_{0.94} \mathrm{NbO}_{3}$ ceramics. Current Applied Physics, 2011, 11: 184-188.

[16] XU HONG-YU, LI QIAN, HUANG JIN-LIANG. Effect of ZnO addition on sintering and dielectric properties of CLST microwave dielectric ceramics. Journal of Functional Materials and Devices, 2008, 14(4): 835-838.

[17] LI YUE-MING, ZHANG BIN, ZHANG HUA, et al. Research on low -temperature sintering of $\mathrm{Ca}_{0.2}\left(\mathrm{Li}_{1 / 2} \mathrm{Sm}_{1 / 2}\right)_{0.8} \mathrm{TiO}_{3}$ microwave dielectric ceramics. Journal of Ceramics, 2010, 31(2): 208-212. 